El discurso de la prensa gráfica sobre el abandono universitario. La educación superior como bien individual Andrés Ignacio Santos Sharpe

http://perio.unlp.edu.ar/ojs/index.php/question/article/view/4693

Cita sugerida: Santos Sharpe, A. (2018). El discurso de la prensa gráfica sobre el abandono universitario. La educación superior como bien individual. Question, 1(59), e077. doi:https://doi.org/10.24215/16696581e077

\title{
El discurso de la prensa gráfica sobre el abandono universitario. La educación superior como bien individual
}

\author{
The discourses of the printed press on university drop out. Higher \\ education as an individual good
}

Andrés Ignacio Santos Sharpe andres_iss@yahoo.com.ar

http://orcid.org/0000-0003-4747-3692

Instituto de Investigaciones Gino Germani; Facultad de

Ciencias Sociales; Universidad de Buenos Aires/

Consejo Nacional de Investigaciones Científicas y Técnicas (Argentina)

\section{Resumen}

El presente artículo analiza discursos de la prensa gráfica sobre el abandono universitario producidos entre 2002 y 2017. Para ello se recuperan notas de los periódicos La Nación y Clarín, debido a que se tratan de los dos diarios de alcance nacional con mayor tirada en la Argentina, con el objeto de dar cuenta de las representaciones compartidas en torno al significante abandono.

Específicamente se trabaja con artículos periodísticos que refieren a los procesos de graduación y abandono, y se indaga sobre el sustrato ideológico observado del corpus analizado en relación a las corrientes de pensamiento en la que se inscribe. Teniendo en cuenta lo dicho, se analizan los recursos argumentativos sobre los cuales construyen el discurso sobre el abandono universitario, particularmente en torno a la idea de bien individual e 
inversión en relación a un mercado laboral. Finalmente, se da cuenta del carácter punitivista asociado al significante abandono.

Palabras clave: educación superior; medios; representación social; deserción.

Abstract

This article analyzes the discourses of the printed press about university abandonment in Argentina during between 2002 and 2017. To this end, we analyze the newspapers La Nación and Clarín, mainly because they have the largest circulation nationwide in Argentina, in order to analyze the shared representations around the significant abandonment.

Specifically, we analyze articles that refer to the processes of graduation and abandonment, and we inquire about the observed ideological substrate of the corpus analyzed in relation to the currents of thought in which it is inscribed. Taking this into account, we analyze the argumentative resources on which the discourse on university dropout is built, particularly around the idea of individual good and investment in relation to a labor market. Finally, it explores the punitive character associated to that discourse.

Keywords: higher education; media; social representations; university drop out.

El presente artículo forma parte de un estudio doctoral en curso (1). La investigación se centra primordialmente el estudio de la experiencia estudiantil por vía de los relatos a efectos de reconstruir las prácticas de los estudiantes en la vida cotidiana. La reconstrucción del sentido que los sujetos le otorgan supone a su vez dar cuenta de "las situaciones biográficas de las personas en su relación con los grupos de pertenencia y referencia y con lo social en su conjunto" (Meccia, 2016: 42).

Considerando lo dicho, el presente artículo se propone analizar el discurso público de uno de los actores dominantes de la escena social en relación al debate sobre el abandono universitario: los medios masivos de comunicación. Analizar el sustrato ideológico de dicho discurso nos permitirá en futuras investigaciones, dar cuenta de cómo opera en la configuración de representaciones sociales sobre la universidad pública y sobre el abandono de los estudios. 
Se parte de la hipótesis de que para comprender las razones que se esgrimen cuando se dejan los estudios universitarios es necesario atender a los discursos públicos y mediáticos que circulan en relación a los procesos de graduación, y cómo se articulan con las distintas concepciones de éxito y fracaso que también encontramos en los relatos de abandono universitario.

De esta manera, la propuesta del presente artículo es retomar los discursos de la prensa gráfica sobre el abandono universitario a partir del análisis de notas en su formato digital. Vale mencionar que el modo en que los periódicos conforman una agenda pública a partir del siglo $X X I$ ya no es el mismo que el de gran parte del siglo $X X$, sin embargo siguen configurando un discurso hegemónico a partir del cual se organizan los debates en otros esferas de lo público como la interacción en redes sociales en el habla cotidiana (Casini, 2007).

Considerando lo previo, se van a analizar artículos entre 2002 y 2017 (2) que abordan el fenómeno del abandono universitario. La elección del recorte se debe principalmente a dos motivos: por un lado, tal como mencionamos previamente, la aparición del periodismo digital exige un análisis diferenciado respecto a cómo eran las dinámicas previas (particularmente a partir del uso extendido de internet que en Argentina supera al 10\% de la población en 2002 (3) y en 2012 pasa a ser el país con mayor uso de internet por persona en la región).

Por otro lado, se eligió el corpus de artículos periodísticos de los últimos 15 años dado que el periodismo dedicado al sistema de educación superior argentino modifica su agenda a partir de 2002: pasó de un predominio en la década del noventa de notas enfocadas en las políticas estatales para con la universidad, a enfocarse en los procesos de masificación, acceso y abandono. Este cambio de la agenda mediática se explica hacia el final del primer apartado.

Recuperando a Pedro Krotsch (2003), "el año 1993 constituye una ruptura con el estilo de política anterior" (2003: 173) en relación a la universidad. Desde ese año hasta 2002 se llevó a cabo una construcción de una agenda de políticas universitarias y su instrumentación desde una perspectiva neoliberal y fuertemente evaluatoria (Krotsch, 2003: 167) en la cual intervenía tanto el gobierno nacional como otros actores claves: el Banco Mundial, en el apoyo ideológico material y operativo; la Secretaría de Políticas Universitarias para operativizar dichas políticas; y los grandes medios en la búsqueda de consenso público para llevar a cabo algunas reformas como la instauración de exámenes de ingreso (Buchbinder y Marquina, 2008).

Sin embargo, a partir de 2002 la agenda mediática sobre educación superior deja de centrarse en esos aspectos por el cambio en la agenda política en relación a las universidades. A partir de entonces, el eje principal de los artículos sobre universidad pasó a centrarse en los procesos de masificación, acceso y abandono. 
De esta manera, para el presente artículo se recuperaron 49 artículos de los diarios La Nación y Clarín debido a que se son los dos periódicos de mayor tirada y de alcance nacional, siendo uno de ellos parte del multimedio más importante en Argentina hasta la fecha, que incluye numerosos canales de televisión, diales radiofónicas, portales de internet y distintos servicios como televisión por cable, conexión a banda ancha entre otros servicios, a partir del cual se organiza una porción significativa de la agenda mediática y cultural.

Dicho corpus se analizará a partir de un breve trabajo arqueológico (Foucault, 1979) que permita visualizar en qué serie discursiva se inscriben estos documentos. En términos de marco enunciativo (Maingueneau, 2003) se trata de estudiar una misma escena englobante (el discurso de la prensa gráfica) en dos escenas genéricas distintas: las notas editoriales y las de especialistas en educación superior.

A modo descriptivo, el corpus está compuesto de cuatro notas editoriales de La Nación; cinco notas sin firma del mismo periódico; dieciocho artículos escritos por distintos autores en ambos diarios, entre ellos periodistas generales, especializados y personalidades invitadas como Alberto Taquini (médico e hijo del académico homónimo quien redactó el Plan Taquini de 1970) y Abraham Gak (contador público de la UBA, ex rector del colegio universitario Carlos Pellegrini e integrante de proyecto Plan Fénix); las restantes veintidós notas fueron firmadas por Alieto Guadagni, contador de la UBA de formación, trabajó como funcionario público desde 1966, actualmente es miembro de la Academia Nacional de Educación y dirige el Centro de Estudios de la Educación Argentina (CEA) perteneciente a la Universidad de Belgrano.

Consideramos necesario dar cuenta de la trayectoria de algunos de los autores en tanto que ocupan una función social de intelectuales públicos (Gramsci, 1984), particularmente del último mencionado. El detalle del corpus y sus autores se encuentra al final del artículo.

En forma preliminar, se pudo observar cómo la construcción discursiva sobre el abandono universitario por parte de los medios masivos mencionados está atravesada por los siguientes ejes:

1. Una concepción individualizada del sujeto.

2. Una perspectiva respecto de la educación, particularizando en el ámbito universitario, en donde prima un énfasis por la eficiencia.

3. Una lógica discursiva que se expresa en cómo presentan "el problema de la educación" y cuáles son sus propuestas para solucionar dicho "problema". 
Uno de los argumentos del presente artículo es que los artículos periodísticos analizados dan cuenta de los ejes mencionados de manera transhistórica y descontextualizadas. En otras palabras, borran sus condiciones de producción.

Considerando lo dicho, el análisis se presenta en el artículo a partir de la siguiente organización: un primer apartado recupera los estudios previos sobre abandono en la Argentina y ubica la tradición teórica en la que se inscriben los discursos de la prensa en relación a dicho fenómeno para así entender su trasfondo teórico-ideológico.

En un segundo apartado comienza con el análisis del corpus de artículos para dar cuenta desde qué lugar se escribieron, cuáles son sus diferencias y similitudes, sus principales argumentos e hipótesis generales con el objetivo de pensarlos en la intimidad de su propia retórica.

Considerando las características del corpus, en un tercer apartado recupera el marco teóricometodológico a partir de la cual se realizó la investigación con el fin de explicar cuál es el sustrato ideológico de los artículos periodísticos están imbricados pero que no se hace explícito. Un cuarto apartado supone el análisis en sí, en donde se buscó comprender este discurso público al ubicarlos en distintas series discursivas. En este apartado se reconstruyen los vínculos, redes y series en las que el corpus se inscribe que dan cuenta de las conclusiones adelantadas.

Finalmente se esbozarán algunos comentarios modo de cierre que harán particular hincapié en cómo estos discursos se presentan a sí mismos como evidencias y se imbrican en la malla social.

\section{Estudios sobre el abandono universitario en una lectura histórica}

Existen distintas concepciones y lecturas respecto al fenómeno del abandono universitario que se explican en su relación con distintas tradiciones teóricas a través de las cuales se percibe y se valora diferencialmente a la universidad. En estudios previos analizamos cuáles fueron las condiciones de emergencia de las investigaciones sobre el fenómeno y sus diferencias en el abordaje del fenómeno tanto a nivel local como internacional (Santos Sharpe, 2016).

Dichos abordajes previos sobre el abandono universitario implican modos distintos de pensar y definir tanto al abandono como al rol de la universidad en relación a la sociedad. Centrándonos en el plano local, la universidad argentina se desarrolló a principios del siglo XX sobre la base 
de una concepción liberal que focalizaba en la responsabilidad del individuo a la hora de explicar las razones del abandono universitario.

La tematización del abandono como una problemática social recién aparece con el surgimiento de la sociología científica entre fines de los cincuenta y principios de los sesenta. Sin embargo, a finales de esa misma década, otra tradición disciplinar empieza a desarrollar explicaciones sobre el abandono: la Teoría del Capital Humano y los estudios econométricos (Santos Sharpe, 2016: 5).

Luego del golpe de estado conocido como la Revolución Argentina, la preocupación de las políticas universitarias estaban más centradas en la creación de la nueva ley para las casas de estudios, la 17.245, los intentos del Poder Ejecutivo constreñir la libertad académica y la autonomía fueron alejando a los investigadores de las ciencias sociales del abandono universitario como un fenómeno prioritario a analizar. Ese vacío respecto al abandono fue recuperado por académicos de las ciencias económicas.

En el marco del andamiaje teórico del Capital Humano, se empezó a pensar al abandono en función de los costos para una sociedad que luego no se ven patentados en un profesional formado; en la "pérdida" de lo que se entiende como una inversión económica. Lo que se puede observar es que en términos de número de investigaciones desarrolladas y de presencia en medios públicos, esta escuela de pensamiento mantiene su hegemonía en la temática.

La década del noventa trajo en sus comienzos nuevos debates y transformaciones en la universidad que fueron configurando una nueva agenda de problemas universitarios (Buchbinder, 2010: 220). Esta agenda se construyó sobre las bases de una crítica a la masificación y a la sobredimensionada crisis presupuestaria de la universidad hacia finales de los ochenta (Buchbinder y Marquina, 2008: 27), situando en un lugar privilegiado los problemas vinculados con "la administración y gestión del presupuesto, las políticas de admisión de los estudiantes, las formas de remuneración del personal docente y no docente, el lugar de la investigación en la Universidad y la conformación de la oferta curricular" (Buchbinder, 2010: 220).

En el mismo sentido, Pedro Krotsch dice que "el año 1993 constituye una ruptura con el estilo de política anterior" (2003: 173) a partir de la cual se "buscó abandonar la tradicional confianza y relación benevolente con la universidad pública" y el accontability se convirtió en el centro del debate al tiempo que se generaba "una nueva agenda sobre la base de los nuevos problemas planteados" (Ibídem).

En la década del noventa, se observa una agenda mediática centralizada en los cambios de la relación estado-universidad y la emergencia de las nuevas políticas que definirían esa relación. 
Las preocupaciones sobre la universidad pública estaban definidas en función de los cambios en materia de políticas universitarias, las orientaciones globales en la educación superior y, en relación a los estudiantes, el movimiento estudiantil en tanto actor protagónico (Carli, 2012). Ese eje sobre los discursos de la prensa comienza a cambiar con las nuevas políticas estatales en relación a la universidad que advinieron a partir del 2003, lo que justifica el período en el que se centra el presente trabajo.

\section{Caracterización temática del corpus y percepciones comunes en torno a la universidad}

Como se especificó en el apartado anterior, recién en las últimas décadas podemos hablar de nuevas investigaciones vinculadas con el abandono universitario en Argentina (Santos Sharpe y Carli, 2016). Resumidamente, se pueden distinguir dos grandes líneas: 1. aquellas investigaciones de la tradición sociológica francesa en las que predomina un énfasis en el análisis de las diferencias culturales y simbólicas que fortalecen formas de desigualdad social y 2. aquellos estudios que desde la economía plantean lecturas que tienen como premisa común que la inversión en conocimiento constituye un factor decisivo para el desarrollo económico. Este concepto de inversión se asume tanto a nivel de gestión estatal como individual, en tanto que los sujetos deciden hacer una inversión económica para darle un plusvalor a su desarrollo personal o de la sociedad.

Sin embargo, estos estudios de principios de la década del noventa se diferenciaban de aquellas primeras investigaciones de los años sesenta. Los primeros "economistas de la educación" de la década del sesenta no tuvieron un peso significativo en los estudios sobre universidad sino hasta fines de los ochenta, cuando fueron releídos y reinterpretados. Como explica Perla Aronson en su artículo "El retorno de la Teoría del Capital Humano" (2007), se encuentran diferencias entre los teóricos del capital humano de los sesenta y de los ochenta, tanto en el carácter predominante que le atribuían al concepto de la educación (2007: 14) como a la concepción del sujeto de la educación (2007: 22).

Esto último se puede observar indagando las preguntas y preocupaciones que esgrimen las notas periodísticas analizadas. La variedad de artículos recopilados ha llevado a sistematizarlos a partir de la construcción de una tipología. Sin dudas la elaboración de una tipología tiene sus peligros dado que hay artículos difíciles de categorizar y matices al interior de cada categoría, pero siendo conscientes de estas limitaciones, se han reconocido cinco grandes ejes temáticos en los que se enfocan todas las notas: 
1. Aquellos que se enfocan en la relación ingresantes/graduados.

2. Los que miran el "costo" que significa para el Estado el mantenimiento de la universidad pública en relación a la cantidad de graduados.

3. Los que construyen un discurso dicotómico entre masividad o calidad.

4. Aquellos que observan cómo el vínculo con el mercado de trabajo abona al abandono.

5. Finalmente, un quinto grupo de artículos son aquellos que hacen una lectura más interesada en las políticas públicas en relación al abandono tanto por parte del Estado y desde la gestión de las universidades, al tiempo que proponen soluciones al abandono o comparan con la situación de otros países.

En general los artículos referidos no se abocan a un solo eje temático, sino que pueden articular los nombrados previamente entre sí o con otros ejes de carácter secundario que vienen a reforzar el eje principal, tales como:

- La relación educación secundaria y universidad.

- La diferenciación entre carreras humanísticas y técnicas.

- La noción de "calidad educativa".

- Los modos específicos de financiamiento de la universidad pública.

- El tiempo que lleva a un estudiante concluir una carrera, entre otros.

Estos cinco ejes secundarios engloban percepciones comunes sobre la universidad por parte de los artículos periodísticos que se podrían resumir de la siguiente manera. Primero, estas perspectivas ideológicas comunes mencionan que los conocimientos y las capacidades humanas se vuelven activos entendidos en términos económicos y, como todo en la esfera del mercado, se convierte en un objeto en el que se invierte y se le busca rentabilidad a lo largo de un período de tiempo. Por ejemplo, el artículo "En universidades públicas: el $44 \%$ aprueba una materia por año" (Polack, 8 de septiembre de 2014) parte de ese dato estadístico presentado en el título para después desarrollar cuál es el costo estimado en dólares por graduado, basándose en una ecuación muy simple: si el Estado otorga $x$ cantidad de pesos por universidad en un período de tiempo, se estima cuántos graduados tiene dicha universidad en ese lapso y el cociente resultante les daría "el costo por graduado".

Un segundo aspecto común es que introducen el fenómeno del abandono universitario a partir de sus supuestos efectos en el mercado laboral, tal como se puede observar en las notas "Los 
graduados sólo cubren el 40\% de la demanda laboral" (Larraquy, 28 de septiembre de 2014) y "En el país se reciben sólo 300 ingenieros cada mil abogados" (Dillón, 7 de junio de 2014), para con ello dar cuenta de la "poca eficiencia" del sistema.

Una tercera perspectiva en común en la que focalizaron estos discursos de la prensa es respecto a las consecuencias que el abandono generaría sobre todo en relación a sus implicancias socio-económicas entendidas como los costos para la sociedad que luego no se verían patentados en un profesional formado, la imposibilidad o dificultad de un planeamiento del mercado de trabajo, entre otros.

Estos artículos, aún con diferencias entre ellos, mantienen una premisa común que se basa en considerar a la educación como un bien de mercado que puede ser econométricamente mensurable y que se debe organizar bajo esos parámetros mercantiles-empresariales.

Tal como se dará cuenta en el próximo apartado, los artículos que componen el corpus analizado presentan una gama problemáticas, preguntas y respuestas que se encuentran todas bajo el paraguas del andamiaje teórico-ideológico de la Teoría del Capital Humano.

\section{Tratamiento del abandono universitario por la prensa}

Las coyunturas político educativas suelen caracterizarse por la circulación amplia de ciertos discursos que condensan una serie más compleja de los modos de apreciar, en este caso, al fenómeno del abandono universitario. Así como también dan cuenta de los horizontes de sentido en los que esas apreciaciones tienden a inscribirse.

Estos discursos y su relación con los procesos históricos en los que se inscriben pueden ser vistos como lugares de condensación de representaciones ideológicas que en este caso se encuentran imbricadas en la mirada de la Teoría del Capital Humano en el campo de la educación. Al mismo tiempo van enlazando formaciones imaginarias que resultan en indicios en la superficie de lo simbólico que se llegan a plasmar, al menos en este caso, en enunciaciones de los medios.

Abocarse al análisis específico de estos artículos periodísticos no supone de ningún modo restringirse a su emergencia literal, ya que bajo distintas formas de enunciación pueden aparecer manifestadas un sistema de remisiones que operan sobre el mismo campo semántico, campo en el que se desarrolla una lucha política. Tal como mencionamos en la introducción, la mirada arqueológica desde la cual se analizan los artículos permite ubicarlos en regímenes de verdad. Dicho de otro modo: el enunciado es un ejemplo tipo en un campo de 
variabilidad, una cristalización que colocamos a modo de referencia, pero es muy probable que los modos de formulación presenten diversas variantes en los materiales objeto de análisis.

Considerando este enfoque metodológico, la elección de las fuentes periodísticas citadas en la introducción del presente artículo se debe a que se tratan de una forma de circulación privilegiada de ciertos discursos que, en el caso del abandono universitario, se caracterizan por seguir la línea de lo economistas de la educación.

Los artículos analizados presentan al abandono universitario como un fenómeno meramente empírico a analizar en una coyuntura particular, lo cual promueve que se considere al fenómeno como ahistórico y sin ideología. Considerando lo dicho, es que es necesario reconstruir la genealogía de dicho discurso. Más allá de su irrupción mediática con el nuevo milenio, sus fundamentos se remontan históricamente hasta principios del siglo XX en donde se pueden apreciar los primeros significantes con las nociones de individuo, inversión y fracaso.

Considerando lo dicho es que este artículo propone dar cuenta cuáles son las prescripciones a las que ciertas formaciones discursivas vinculadas con el abandono están interrelacionadas. Esto supone, por un lado localizar las condiciones de posibilidad que hacen que el discurso sobre el abandono universitario se articule en torno a: una idea de sujeto como individuo, una construcción de la educación como inversión, una relación unidireccional entre graduado y mercado del trabajo, una noción de abandono ligada a un imaginario de fracaso y de costo para la sociedad, y una organización de la universidad en torno a la lógica de la eficiencia.

Estas formaciones discursivas (educación como inversión, el sujeto como individuo, abandono como fracaso o gasto y preeminencia de la eficiencia económica) se materializan en acciones de los sujetos expresadas como creencias en un discurso en apariencia sin fisuras, coherente que expresa, condensa y articula múltiples enunciaciones e interpretaciones sobre la construcción del fenómeno analizado, que exceden a la enunciación textual de cada frase y remite a un campo semántico más amplio sobre la visión del país, la economía, la sociedad, la historia y por supuesto a la posición de quien la enuncia.

Pensando este discurso de la prensa que se desarrolló en el apartado anterior, podemos preguntarnos: ¿qué representación de estudiante organiza?, ¿qué visión de cómo ejercer el conocimiento aprehendido? Ahí se observa que la graduación universitaria se pondera como un fin en sí mismo, así como la concepción de "continuar los estudios" luego de la escolaridad secundaria con el fin de acceder a un trabajo calificado mejor pago. 
De esta manera, el abandono termina asociado al significante del fracaso y de la estigmatización social por el costo que ese sujeto pensado como individuo resultó para su familia, para la sociedad, para la institución universitaria y para él mismo.

\section{La fabricación de la consecuencia: un análisis de los recursos argumentativos sobre el abandono universitario}

La formación discursiva analizada en los artículos periodísticos se presenta a la sociedad bajo un discurso que se muestra preocupada por los sectores de menor nivel socio-económico, siendo el argumento que construyen el siguiente: toda la sociedad (incluyendo los quintiles inferiores) mantiene a la universidad pública a través de sus impuestos, de cuyas aulas solo se gradúan el $27 \%$ y, principalmente, sólo aquellos provenientes de los niveles socio-económicos medios de la sociedad (tal como lo expresa la nota de La Nación del 4 de junio de 2013). El falso silogismo que luego presentan es: si solo terminan egresando aquellos que a priori tienen ingresos familiares suficientes para pagar la universidad, entonces arancelemos la universidad y frenemos esta injusticia en la cual los pobres mantienen a los sectores más acaudalados.

De esta manera, como menciona Teun Van Dijk, el discurso periodístico se valoriza en función de la seriedad con la que presentan sus consecuencias. Es "mediante la discusión real posible de las consecuencias, un discurso periodístico puede otorgar coherencia causal a los acontecimientos informativos" (1990: 85). En función de ello es que el autor neerlandés entiende que en el discurso periodístico "las consecuencias son incluso más importantes que los propios acontecimientos informativos principales" (Ibídem).

En el mismo sentido, el discurso mediático aquí recuperado se centra en las consecuencias que el abandono tendría para la sociedad. Dicha lógica discursiva se observa no solo en las notas periodísticas, sino también en los trabajos que Alieto Guadagni, una de las figuras públicas que impulsan este discurso tanto desde los medios masivos y como en boletines académicos, redactó:

(...) es importante tener presente que una universidad con pocos graduados en proporción al total de alumnos incrementa fuertemente el costo presupuestario de cada graduado a cargo de los contribuyentes. (...) No hay que olvidar que el financiamiento de la universidad proviene de millones de argentinos que no pueden acceder a ella, porque no concluyen la escuela secundaria y la inmensa mayoría de ellos son pobres (Guadagni, 2014: 11). 
Desde esta perspectiva se sugiere que hay una decisión razonada a partir de toda la información disponible en el mercado desde la cual el agente económico (pensado como individuo) toma la decisión de invertir o no en su educación y de seguir estudiando o abandonar, en donde el sujeto sopesa entre los costos de inversión (que incluye el tiempo que le demande estudiar de acuerdo a sus capacidades) en relación a los beneficios que obtendrá en el futuro si sigue formándose. Por ejemplo, la columna editorial de La Nación del 27 de octubre de 2014 se pregunta:

Que un estudiante goce de ventajas en un sistema de estudios cuyas reglas fundamentales incumple pareciera ser un argumento absolutamente inconsistente y disparatado. (...) ¿Cómo sostener que quienes sí respetan los planes de estudio deban recibir una educación condicionada o acotada por una inversión inútil? ¿Cómo programar un gasto racional y optimizar sus beneficios frente a una matrícula de estudiantes que jamás completará su educación más que en un ínfimo porcentaje? (La Nación, 27 de octubre de 2014).

Se puede observar en la cita el lugar que se le asigna al sujeto entendido como individuo atomizado que toma decisiones tales como abandonar o continuar sus estudios superiores fundadas en un supuesto criterio consciente y que dependen totalmente de sí mismo. Sin embargo, esta supuesta racionalidad de los actores en relación a las decisiones que toman, no las recuperan sobre lo dicho por los propios actores, sino que las infieren a partir de las estadísticas sobre deserción.

Encontramos sin embargo algunas disidencias entre los distintos autores en las propuestas para solucionarlo (como el caso del artículo de Abraham Gak, quien apoya el ingreso irrestricto y menciona la necesidad de no cargar de culpas a la escuela media sino que la universidad asuma la responsabilidad del "fracaso" estudiantil). A pesar de ello, predominan los elementos en común en torno al imaginario construido por los discursos de la prensa sobre el abandono universitario que resumimos en los siguientes cinco puntos:

1. En primera instancia, asumen a la enseñanza en el nivel superior y a la producción científica y académica que allí se realiza como una inversión. Inversión que resulta indispensable para el desarrollo económico (o al menos para cierta forma de desarrollo económico).

2. Esa inversión, siguiendo la lógica de los discursos públicos y mediáticos analizados, la debe hacer el individuo (presentado en genérico) quien previamente hace una estimación de sus posibilidades de graduarse y el rédito económico que eso le supone. Esto construye discursivamente a la educación como bien individual, no social. 
3. En este sentido, la educación es presentada únicamente como un objeto a ser pensada en función del mercado laboral.

4. Por otro lado, mantienen como premisa que el individuo arbitra racionalmente y conoce el amplio abanico de opciones posibles para tomar decisiones.

5. Consecuentemente, la lógica a partir de la cual presentan el abandono es presentando al mismo como una inversión desperdiciada y en relación a sus supuestas implicancias socioeconómicas (por ejemplo, los altos costos para la sociedad que luego no se ven patentados en un profesional formado).

La pregunta por cómo racionalizar el gasto da por supuesto que la educación es un gasto más y presupone que el principal "producto" de la universidad es el generar graduados, ignorando otras cuestiones como la producción científica o la extensión a la sociedad. Pero también deja de lado fenómenos no cuantificables como el hecho de que la universidad es un espacio de conformación de subjetividades a partir de las cuales los sujetos (graduados, estudiantes o quienes hayan dejado los estudios) se relacionan con el mundo de una manera diferenciada respecto de aquellos que nunca transitaron sus aulas. En otras palabras, según esta perspectiva, el problema no es el abandono, ni los sujetos que abandonan, sino únicamente el costo económico que el abandono generaría.

Sumado a lo anterior, podemos observar en la bajada de la nota de Clarín "Los graduados sólo cubren el $40 \%$ de la demanda laboral" lo siguiente: "El Estado les paga para que se reciban. Perciben los mejores salarios del mercado y pueden decidir dónde trabajar. Sin embargo, la vocación tecnológica sigue siendo baja para las necesidades del país" (Larraquy, 28 de septiembre de 2014).

"El estado les paga para que se reciban" denota una concepción deudora del sujeto al estado que "le paga" sus estudios superiores. Una obligatoriedad del estudiante, basada en una concepción que se podría resumir de la siguiente manera: yo te pago para que hagas esto, ergo es tu obligación hacerlo. Según la lógica discursiva acá presentada, el estudiante debe finalizar sus estudios porque el estado paga y así se esquematiza y resume todo el fenómeno a una "relación costo-egresado" (Polack, 8 de septiembre de 2014).

Lo que se puede entrever en los artículos analizados es que todo el fenómeno del abandono universitario se reduce eminentemente al costo que tiene para el estado mantener la gratuidad en la educación superior; proponiendo un enfoque "eficientista" de la educación.

Sin embargo, es importante destacar que para que esa operación sea efectiva, el fenómeno de la educación tiene que ser presentado de manera transhistórica y descontextualizadamente, 
instalando el discurso de la educación como una inversión y la igualación del abandono al fracaso como una evidencia, fortaleciendo así las prácticas competitivistas, evaluatorias, y confiscatorias de la educación y las competencias de empleabilidad y selección fundadas en habilidades y resultados.

\section{A modo de cierre}

En el presente artículo se buscó dar cuenta de cuáles eran los discursos de la prensa gráfica respecto del abandono universitario, cómo era la construcción de ese discurso y a qué formaciones ideológicas se correspondían.

De esta manera se pudo observar una formación discursiva sobre el abandono universitario dentro de la perspectiva del Capital Humano. Se presentaba al abandono como un problema que "perjudica" a toda la sociedad. Dicho vínculo entre fenómeno del abandono y "la sociedad" (como entidad discursiva en esa formación) no se realiza desde cualquier lugar o perspectiva: pesa una percepción punitiva sobre el abandono que redituaría en pérdidas económicas para la sociedad que "mantiene a través de sus impuestos" (La Nación, 27 de octubre de 2014) a todos los estudiantes.

A lo largo del trabajo se pudo localizar las condiciones de posibilidad que hacen que el discurso sobre el abandono universitario se articule en torno a una idea de sujeto como individuo, una construcción de la educación como inversión, un vínculo estrecho entre graduado y mercado del trabajo y una noción de abandono ligada a un imaginario de fracaso y de costo para la sociedad.

Paralelamente, el discurso de la Teoría del Capital Humano sobre el abandono hace un fuerte eje en la asociación de la deserción con el fracaso y cómo dicho fracaso redituaría en un mayor costo en el sistema público universitario argentino, lo cual termina siendo un discurso que reduce a la universidad únicamente a la producción de graduados.

A partir de ese argumento, surgen las propuestas de "volver más eficiente" a la universidad instituyendo exámenes de ingreso que funcionen como "filtro" previo, para evitar a la sociedad el gasto de tener tantos estudiantes que luego abandonan, entre otras propuestas tendientes a ciertas formas de arancelamiento del sistema educativo superior.

Estas articulaciones resultan de lo que Foucault denomina una práctica discursiva específica. En este sentido, resulta primordial preguntarse por el proceso de conformación de esta forma de representarse el mundo. La cual opera tanto para el estudiante que finaliza la cursada 
regular de una carrera, que se piensa como exitoso; para el estudiante que abandona; para su familia, que entiende ese tránsito como una pérdida de tiempo; y, en general, en los discursos públicos, mediáticos y sociales que, sin tantas mediaciones, aducen esta situación como una pérdida de inversión pasible de ser trasladadas a otras áreas en donde sean mejor aprovechadas.

Se observa entonces que esta consolidación de una discursividad pública sobre el abandono universitario en torno al significante de fracaso tiene consecuencias mucho más amplias que las estrictamente referidas al sujeto que abandona una carrera universitaria.

Cabe preguntarse entonces, en futuros trabajos, cuáles son los aprendizajes que tienen los sujetos en su tránsito por la universidad más allá de la inconclusión de una carrera que permitirían dar cuenta de un mundo más allá de los estándares de eficiencia.

\section{Notas}

(1) El presente artículo se inscribe en el marco de una beca de doctorado CONICET dirigida por Evaluación-anónima, en la que se procura indagar sobre las experiencias de jóvenes estudiantes que hayan discontinuado su educación superior en la Universidad de Buenos Aires (UBA).

(2) Si bien todos los artículos recopilados no aparecerán citados en el presente artículo, forman parte del corpus que dio cuerpo a esta investigación y aparecen en el corpus hemerográfico.

(3) Las estadísticas por país y por año están disponibles online en http://www.itu.int/en/lTUD/Statistics/Pages/stat/default.aspx visto el 6 de julio de 2017.

\section{Corpus hemerográfico}

Bilinkis, S. (20 de diciembre de 2015). ¿Qué elegimos estudiar? La Nación. Recuperado de http://www.lanacion.com.ar/1855560-que-elegimos-estudiar

Carrizo, C. (31 de octubre de 2014). Más universidades, pero menos calidad educativa. La Nación. Recuperado de http://www.lanacion.com.ar/1740065-mas-universidades-peromenos-calidad-educativa

Dillón, A. (7 de junio de 2014). En el país se reciben sólo 300 ingenieros cada mil abogados. Clarín. Recuperado de http://www.clarin.com/sociedad/pais-reciben-solo-ingenierosabogados_0_1170482991.html 
Editorial (3 de abril de 2016). Empleabilidad de los graduados universitarios. La Nación. Recuperado de http://www.lanacion.com.ar/1885641-empleabilidad-de-los-graduadosuniversitarios

Editorial. (28 de julio de 2014). Cuando la educación deja de ser la meta. La Nación. Recuperado de http://www.lanacion.com.ar/1713567-cuando-la-educacion-deja-de-serla-meta

Editorial. (27 de octubre de 2014). El viejo y falso mito de la gratuidad. La Nación. Recuperado de http://www.lanacion.com.ar/1738941-el-viejo-y-falso-mito-de-la-gratuidad

Editorial. (30 de noviembre de 2015). Educación sin fronteras. La Nación. Recuperado de http://www.lanacion.com.ar/1849962-educacion-sin-fronteras

Gak, A. (16 de febrero de 1998). Uno de cada tres no aprueba el CBC. La Nación. Recuperado de http://www.lanacion.com.ar/88098-uno-de-cada-tres-no-aprueba-el-cbc

Gil, V. (19 de febrero de 1998). Más resultados adversos en la universidad platense. La Nación. Recuperado de http://www.lanacion.com.ar/88340-mas-resultados-adversos-en-launiversidad-platense

Guadagni, A. (13 de agosto de 1999). Cada graduado le cuesta al Estado \$44.863. La Nación. Recuperado de http://www.lanacion.com.ar/149395-cada-graduado-le-cuesta-al-estado44863

Guadagni, A. (1 de octubre de 2000). El derroche universitario. La Nación. Recuperado de http://www.lanacion.com.ar/35109-el-derroche-universitario

Guadagni, A. (31 de octubre de 2000). Equidad en el gasto educativo. La Nación. Recuperado de http://www.lanacion.com.ar/39061-equidad-en-el-gasto-educativo

Guadagni, A. (24 de agosto de 2000). Función de la universidad. La Nación. Recuperado de http://www.lanacion.com.ar/30140-funcion-de-la-universidad

Guadagni, A. (17 de julio de 2001). Calidad educativa. La Nación. Recuperado de http://www.lanacion.com.ar/320388-calidad-educativa

Guadagni, A. (14 de marzo de 2003). Educación, clave del siglo XXI. La Nación. Recuperado de http://www.lanacion.com.ar/480515-educacion-clave-del-siglo-xxi

Guadagni, A. (3 de junio de 2004). Construyamos el futuro. La Nación. Recuperado de http://www.lanacion.com.ar/606796-construyamos-el-futuro

Guadagni, A. (25 de noviembre de 2008). ¿Qué universidad queremos? La Nación. Recuperado de http://www.lanacion.com.ar/1073803-que-universidad-queremos

Guadagni, A. (19 de agosto de 2009). Otra universidad para el futuro. La Nación. Recuperado de http://www.lanacion.com.ar/1163938-otra-universidad-para-el-futuro 
Guadagni, A. (20 de septiembre de 2010). A contramano del mundo. La Nación. Recuperado de http://www.lanacion.com.ar/1306315-a-contramano-del-mundo

Guadagni, A. (26 de septiembre de 2011). ¿Quién paga la universidad? La Nación. Recuperado de http://www.lanacion.com.ar/1409228-quien-paga-la-universidad

Guadagni, A. (7 de septiembre de 2011). El siglo de la calidad. La Nación. Recuperado de http://www.lanacion.com.ar/1403992-el-siglo-de-la-calidad

Guadagni, A. (20 de junio de 2012). En educación, aprendamos de Brasil. La Nación. Recuperado de http://www.lanacion.com.ar/1483533-en-educacion-aprendamos-debrasil

Guadagni, A. (8 de febrero de 2013). Inclusión social para la UBA. La Nación. Recuperado de http://www.lanacion.com.ar/1552785-inclusion-social-para-la-uba

Guadagni, A. (16 de septiembre de 2013). No estamos ayudando a nuestros estudiantes. La Nación. Recuperado de http://www.lanacion.com.ar/1620161-no-estamos-ayudando-anuestros-estudiantes

Guadagni, A. (25 de julio de 2014). Hay que fortalecer la universidad. La Nación. Recuperado de http://www.lanacion.com.ar/1712747-hay-que-fortalecer-la-universidad

Guadagni, A. (8 de febrero de 2014). Igualdad de oportunidades en la universidad. La Nación. Recuperado de http://www.lanacion.com.ar/1662336-igualdad-de-oportunidades-en-launiversidad

Guadagni, A. (14 de enero de 2014). Una universidad para el siglo XXI. La Nación. Recuperado de http://www.lanacion.com.ar/1655309-una-universidad-para-el-siglo-xxi

Guadagni, A. (10 de septiembre de 2015). Educación superior: Aprender de Brasil. Clarín. Recuperado de http://www.clarin.com/opinion/Universidad-Examen_de_IngresoGraduacion-Secundaria_0_1428457188.html

Guadagni, A. (28 de diciembre de 2015). En educación, hay que premiar el esfuerzo. La Nación. Recuperado de http://www.lanacion.com.ar/1857578-en-educacion-hay-quepremiar-el-esfuerzo

Guadagni, A. (6 de abril de 2016). No consolidemos el retroceso educativo. La Nación. Recuperado de http://www.lanacion.com.ar/1886475-no-consolidemos-el-retrocesoeducativo

Guadagni, A. (7 de marzo de 2016). Nuestra universidad desperdicia recursos. Clarín. Recuperado de http://www.clarin.com/opinion/universidad-desperdiciarecursos_0_1535846760.html 
La Nación, (4 de diciembre de 2011). Apoyo psicológico para evitar la deserción. Recuperado de http://www.lanacion.com.ar/1430021-apoyo-psicologico-para-evitar-la-desercion

La Nación, (21 de noviembre de 2014). Las universidades nacionales más nuevas serán las más beneficiadas por el presupuesto. Recuperado de http://www.lanacion.com.ar/1745717-las-universidades-nacionales-mas-nuevas-seranlas-mas-beneficiadas-por-el-presupuesto

La Nación, (26 de febrero de 2015). Por primera vez, hay más inscriptos en la Facultad de Ingeniería que en la de Sociales. Recuperado de http://www.lanacion.com.ar/1771598por-primera-vez-hay-mas-inscriptos-en-la-facultad-de-ingenieria-que-en-la-de-sociales

Larraquy, M. (28 de septiembre de 2014). Los graduados solo cubren el $40 \%$ de la demanda laboral. Clarín. Recuperado de http://www.clarin.com/edicion-impresa/graduados-solocubren-demanda-laboral_0_1220278040.html

Leguizamón, M. \& Beremblum, F. (4 de octubre de 2014). El desafiante pasaje de la secundaria a la Universidad. La Nación. Recuperado de http://www.lanacion.com.ar/1732545-eldesafiante-pasaje-de-la-secundaria-a-la-universidad

Lutvak, A. (12 de mayo de 2015). Repensar el CBC para que sea formativo. La Nación. Recuperado de http://www.lanacion.com.ar/1792039-repensar-el-cbc-para-que-seaformativo

Manzoni, C. (9 de octubre de 2011). Los títulos universitarios le cuestan al Estado cada vez más. La Nación. Recuperado de http://www.lanacion.com.ar/1412982-los-titulosuniversitarios-le-cuestan-al-estado-cada-vez-mas

Masoero, H. (3 de marzo de 2016). ¿Gratuidad universitaria es sinónimo de igualdad? La Nación. Recuperado de http://www.lanacion.com.ar/1876121-gratuidad-universitaria-essinonimo-de-igualdad

Massa, F. (27 de marzo de 2015). Más matriculados, pero pocos graduados en las universidades. La Nación. Recuperado de http://www.lanacion.com.ar/1779480-masmatriculados-pero-pocos-graduados-en-las-universidades

Polack, M. E. (8 de septiembre de 2014). En universidades públicas el $44 \%$ aprueba una materia por año. La Nación. Recuperado de http://www.lanacion.com.ar/1725369-enuniversidades-publicas-el-44-de-los-alumnos-aprueba-una-materia-por-anous-60000

Premat, S. (24 de febrero de 2011). Pocos terminan la universidad. La Nación. Recuperado de http://www.lanacion.com.ar/1352491-pocos-terminan-la-universidad 
Ríos, S. (23 de octubre de 2014). Becas: El secreto del desarrollo universitario uruguayo. La Nación. Recuperado de http://www.lanacion.com.ar/1737755-becas-el-secreto-deldesarrollo-universitario-uruguayo

Rolón, L. (31 de octubre de 2015). Prohíben el examen de ingreso y hay polémica. La Nación. Recuperado de http://www.lanacion.com.ar/1841519-prohiben-el-examen-de-ingreso-yhay-polemica

Sánchez, F. (4 de junio de 2013). Universidades. Se reciben sólo 27 alumnos de cada 100 que ingresan. La Nación. Recuperado de http://www.lanacion.com.ar/1588252universidades-se-reciben-solo-27-alumnos-de-cada-100-que-ingresan

Taquini, A. (11 de noviembre de 2015). Impulsar la educación superior. La Nación. Recuperado de http://www.lanacion.com.ar/1844273-impulsar-la-educacion-superior

Vázquez, L. (18 de marzo de 2016). Educación. ¿Vale la pena ir a la universidad? La Nación. Recuperado de http://www.lanacion.com.ar/1880841-educacion-vale-la-pena-estudiaruna-carrera-en-la-universidad

\section{Bibliografía}

Aronson, P. (2007). El retorno de la teoría del capital humano. Fundamentos en humanidades, VIII(II). Universidad Nacional de San Luis, Argentina.

Buchbinder, P. (2010). Historia de las universidades argentinas. Buenos Aires, Sudamericana, 2010.

Buchbinder, P. y Marquina, M. (2008). Masividad, heterogeneidad y fragmentación: el sistema universitario argentino 1983-2007. Los Polvorines: Univ. Nacional de General Sarmiento; Buenos Aires: Biblioteca Nacional

Carli, S. (2012). El estudiante universitario. Hacia una historia del presente de la educación pública. Buenos Aires, Siglo XXI.

Casini, J. (2007) Periodismo 3.0: el ciudadano como periodista. Recuperado de http://sedici.unlp.edu.ar/bitstream/handle/10915/45563/Documento_completo_.pdf?se quence $=1$

Foucault, M. (1979). La arqueología del saber. México: Siglo XXI editores.

Gramsci, A. (1984). Los intelectuales y la organización de la cultura. Trad. de Raúl Sciarreta. Buenos Aires: Nueva Visión. 
Guadagni, A. (2014). Las universidades nacionales deben cuidar mejor sus recursos. Boletín CEA, 19. Buenos Aires, Universidad de Belgrano.

Krotsch, P. (2003). Educación superior y reformas comparadas. Buenos Aires: Universidad Nacional de Quilmes.

Maingueneau, D. (2003): ¿ «Situación de enunciación» o «situación de comunicación»? Discurso.org, 2(5).

Meccia, E. (2016). El tiempo no para: los últimos homosexuales cuentan la historia. Santa Fe: Ediciones UNL; Buenos Aires: Eudeba.

Santos Sharpe, A. (2016). La discontinuidad de los estudios superiores. Un análisis histórico del abordaje sobre el abandono universitario. Anuario de Historia de la Educación, 17(2), pp. 3-31. Recuperado de http://ppct.caicyt.gov.ar/index.php/anuario/article/view/7722/pdf

Santos Sharpe, A. y Carli, S. (2016). Estudios globales y locales sobre el abandono de los estudios universitarios. Teorías, perspectivas y nuevos abordajes. RAES Revista Argentina de Educación Superior, 8(13), noviembre. Recuperado de http://www.revistaraes.net/revistas/raes13_art1.pdf

Van Dijk, T. (1990). La noticia como discurso. Barcelona: Paidós. 